\title{
Blocking Sets in the complement of hyperplane arrangements in projective space
}

\author{
S. Settepanella *
}

January 2008

\begin{abstract}
It is well know that the theory of minimal blocking sets is studied by several author. Another theory which is also studied by a large number of researchers is the theory of hyperplane arrangements. We can remark that the affine space $A G(n, q)$ is the complement of the line at infinity in $P G(n, q)$. Then $A G(n, q)$ can be regarded as the complement of an hyperplane arrangement in $P G(n, q)$ ! Therefore the study of blocking sets in the affine space $A G(n, q)$ is simply the study of blocking sets in the complement of a finite arrangement in $P G(n, q)$. In this paper the author generalizes this remark starting to study the problem of existence of blocking sets in the complement of a given hyperplane arrangement in $P G(n, q)$. As an example she solves the problem for the case of braid arrangement. Moreover she poses significant questions on this new and interesting problem.
\end{abstract}

\section{Introduction}

Throughout this paper, $P G(n, q)$ and $A G(n, q)$ will respectively denote the ndimensional projective and affine space over the finite field $G F(q)$.

A t-blocking set $B$ in $P G(n, q)$ (or $A G(n, q)$ ) is a set $B$ of points such that any $(n-t)$-dimensional subspace intersects $B$. A 1-blocking set is simply called a blocking set. A $t$-blocking set is called minimal, if none of its proper subsets is also a $t$-blocking set.

The smallest $t$-blocking sets have been characterized by Bose and Burton 2. They proved that the smallest $t$-blocking sets in $P G(n, q)$ are subspace of dimension $t$. An old result of Bruen 3 states that the second smallest minimal blocking set in the plane $P G(2, \sqrt{q}), q$ a square, is (the point set) of a Baer subplane $P G(2, \sqrt{q})$.

There are several survey papers about blocking sets (see Szõnyi, Gács, Weiner [6] and chapter 13 of the second edition of Hirschfeld's book [7]). Most of the surveys concentrate on small minimal blocking sets. But there are also several results about large minimal blocking sets. The first such result is due to Bruen and Thas [4. More results on the spectrum of minimal blocking sets in planes of small order can be found in Cossidente, Gács et alt [5] Innamorati [8],Inamorati and Maturo 9 .

\footnotetext{
*Dipartimento di Scienze della Comunicazione,Colle Parco, 64100 Teramo, Italy
} 
Another interesting problem is the existence of minimal Blocking sets in $P G(n, q)$ and $A G(n, q)$ for fixed $n$ and $q$ (see Mazzocca-Tallini [10] and BeutelspacherEugeni [1).

It is well know that the theory of minimal blocking sets is studied by several author. There is another theory which is also studied by a large number of researchers: the theory of hyperplane arrangements (see the Orlik-Terao's book [11] for a survey on the theory).

An hyperplane arrangement is a set of hyperplane in a given space.

The author remarks that the affine space $A G(n, q)$ is the complement of the line at infinity in $P G(n, q)$. Then $A G(n, q)$ can be regarded as the complement of an hyperplane arrangement in $P G(n, q)$ !

Therefore the study of blocking sets in the affine space $A G(n, q)$ is simply the study of blocking sets in the complement of a finite arrangement in $P G(n, q)$.

The author generalized this remark studying the problem of the existence of blocking sets in the complement of a given hyperplane arrangement in $P G(n, q)$.

In this paper author introduces the first results on this new theory, she generalizes some results of the old theory to the new one.

Moreover she solves the problem of existence of blocking sets in the complement of a well-known and studied arrangement: the braid arrangement, the set of reflection hyperplanes generated by the symmetric group.

She concludes the paper posing several questions:

1. on the existence of blocking sets on the complement of hyperplane arrangements;

2. on the characterization of hyperplane arrangements in $P G(n, q)$ which give rise to interesting new problem on blocking sets;

3. on the link between the two theories.

Clearly also the problem of small and large blocking sets can be studied again in the complement of a given hyperplane arrangement. The author is still working to this problem.

Plainly in this paper author writes blocking sets instead of minimal blocking sets.

\section{General results on Blocking sets}

Let $P G(n, q)(A G(n, q))$ the n-dimensional projective (affine) space over a finite field $G F(q)$. We have the following:

Proposition 1 Let $C$ be a blocking set in $P G(n, q)(A G(n, q))$ with $n>2$ and $S_{d}$ a subspace of dimension $d>1$, then the intersection $C \cap S_{d}$ is a blocking set in $S_{d}$.

Proposition 2 Let $S_{n-1}$ an hyperplane in $P G(n, q)(n>2)$. If $C_{1}$ is a blocking set in $A G(n, q)=P G(n, q) \backslash S_{r-1}$ and $C_{2}$ is a blocking set in $S_{r-1}$, then $C_{1} \cup C_{2}$ is a blocking set in $P G(n, q)$.

Corollary 1 If $P G(n, q)$ and $A G(n+1, q)$ contain blocking sets then also $\mathbf{P G}(r+$ $1, q)$ does. 
The proof of the above statements is trivial.

Mazzocca and Tallini in [10] proved the following:

Theorem 1 There is a function $b_{p}(t, q)\left(b_{a}(t, q)\right)$, depending on $t$ and $q$, such that $P G(n, q)(A G(n, q))$ contains $t$-blocking sets if and only if $r \leq b_{p}(t, q)$ $\left(r \leq b_{a}(t, q)\right)$.

Another interesting general result on existence of blocking set is the following:

Theorem 2 (A. Beutelspacher-F. Eugeni [1]). Let $P G(n, q)$ and $A G(n, q)$ be the projective and affine $n$-dimensional space over a finite field $G F(q)$. If $q \geq 2^{n}$ then $A G(n, q)$ and $P G(n, q)$ contains t-blocking sets.

\section{Blocking sets on the complement of hyper- plane arrangements in $\mathrm{PG}(\mathrm{n}, \mathrm{q})$}

In this section we generalize some interesting results on Blocking Sets in $P G(n, q)$ to the complement of hyperplane arrangements.

Let $\mathcal{A}=\left\{H_{1}, \ldots, H_{m}\right\}$ be an arrangement of hyperplanes in $P G(n, q)$ and

$$
M(\mathcal{A})=P G(n, q) \backslash \cup_{i=1, \ldots m} H_{i}
$$

be the complement of the arrangement.

Let $\mathcal{A}_{a}=\left\{H_{1}, \ldots, H_{m}\right\}$ be an arrangement in $A G(n, q)$ and

$$
M_{a}(\mathcal{A})=A G(n, q) \backslash \cup_{i=1, \ldots m} H_{i}
$$

be the complement in the affine space.

Given an arrangement $\mathcal{A}$ in $P G(n, q)(A G(n, q))$, the corresponding arrangement in $P G(k, q)(A G(k, q))$ for $k \neq n$ is the arrangement given by hyperplanes with the same equations of those in $\mathcal{A}$. From now on we will also use $\mathcal{A}$ to refer to corresponding arrangements of $\mathcal{A}$.

Let us remarks that the statements of propositions 1, 2 and corollary 1 above hold trivially also for Blocking sets in the complements $M(\mathcal{A}) \subset P G(n, q)$ and $M_{a}(\mathcal{A}) \subset A G(n, q)$.

Moreover we have the generalization of Mazzocca-Tallini's theorem:

Theorem 3 Let $M(\mathcal{A})$ be the complement of an arrangement $\mathcal{A}$ in the projective (affine) space $P G(n, q)(A G(n, q))$. Then we can find a function $b_{p, \mathcal{A}}(t, q)$ $\left(b_{a, \mathcal{A}}(t, q)\right)$, depending on $t$ and $q$, such that $M(\mathcal{A}) \subset P G(n, q)(M(\mathcal{A}) \subset$ $A G(n, q))$ has $t$-blocking sets if and only if $r \leq b_{p, \mathcal{A}}(t, q)\left(r \leq b_{a, \mathcal{A}}(t, q)\right)$.

Proof Let $d_{M(\mathcal{A}(n, q))}$ be the maximum of the dimensions of linear subspaces in $M(\mathcal{A}(n, q))$.

Then, by simple geometric consideration, we remark that $\left\{d_{M(\mathcal{A}(n, q))}: n \in\right.$ $\mathbb{N}\}$ is an increasing function.

Let us suppose that $\left\{r_{n}: n \in \mathbb{N}\right\}$ is a sequence of integer such that for all $r_{n}$ there is an $h$-blocking set $B\left(r_{n}\right)$ in $M(\mathcal{A}(n, q))$.

Then for $d_{M\left(\mathcal{A}\left(r_{n}, q\right)\right)}>h$ let $B\left(r_{n}\right) \cap S\left(r_{n}\right)$ be the intersection between $B\left(r_{n}\right)$ and a linear variety $S\left(r_{n}\right)$ in $M\left(\mathcal{A}\left(r_{n}, q\right)\right)$ of dimension $d_{M\left(\mathcal{A}\left(r_{n}, q\right)\right)}$. Then 
$B\left(r_{n}\right) \cap S\left(r_{n}\right)$ is an $h$-blocking set in $S\left(r_{n}\right)$, but this contradicts theorem 1,

\section{The braid arrangement}

Let us consider the Braid arrangement

$$
\mathcal{A}\left(A_{n, q}\right)=\left\{H_{i, j}\right\}_{1 \leq i<j \leq n+1}
$$

given by reflection hyperplanes $H_{i, j}$ in $P G(n+1, q)$ of equations $\alpha_{i, j}: x_{i}-x_{j}=0$.

The complement $M\left(\mathcal{A}\left(A_{n, q}\right)\right)$ is defined by points

$$
\left(x_{0}, x_{1}, \ldots, x_{n+1}\right) \in P G(n+1, q)
$$

such that $x_{i} \neq x_{j}, 1 \leq i<j \leq n+1$. Then we have the following:

Proposition 3 The complement $M\left(\mathcal{A}\left(A_{n, q}\right)\right)$ contains Blocking sets for all $n \leq$ $q-1$ and it is empty otherwise.

In order to prove proposition 3 we need the following lemmas:

Lemma 1 For any two points $\underline{x}$ and $\underline{y}$ in the complement $M_{a}\left(\mathcal{A}\left(A_{q, q}\right)\right)$, the line

$$
\underline{y}+t(\underline{x}-\underline{y})
$$

is in the complement iff $\underline{x}-\underline{y}=(1, \ldots, 1)$. In particular $M_{a}\left(\mathcal{A}\left(A_{q, q}\right)\right)$ contains $(q-1)$ ! lines.

Proof.Clearly $M_{a}\left(\mathcal{A}\left(A_{q, q}\right)\right)$ contains all lines trough a point $P \in M_{a}\left(\mathcal{A}\left(A_{q, q}\right)\right)$ and with direction $(1, \ldots, 1)$.

By contrary let $\underline{x}, \underline{y} \in M_{a}\left(\mathcal{A}\left(A_{q, q}\right)\right)$ be such that $\underline{x}-\underline{y} \neq(1, \ldots, 1)$. If $\underline{x}=\left(x_{1}, \ldots, x_{q+1}\right)$ and $\underline{y}=\left(y_{1}, \ldots, y_{q+1}\right)$ then there exists $i, j$ such that $x_{i}-y_{i} \neq$ $x_{j}-y_{j}$. Let define:

$$
t_{0}=\frac{\left(y_{j}-y_{i}\right)}{\left(x_{i}-y_{i}\right)-\left(x_{j}-y_{j}\right)}
$$

then $t_{0} \neq 0$. Moreover, if $P_{i}$ and $P_{j}$ are, respectively, the $i$-th and the $j$-th entries of $P=\underline{y}+t_{0}(\underline{x}-\underline{y})$, then $P_{i}=P_{j}=\frac{x_{i} y_{j}-x_{j} y_{i}}{\left(x_{i}-y_{i}\right)-\left(x_{j}-y_{j}\right)}$, i.e. $P \notin M_{a}\left(\mathcal{A}\left(A_{q, q}\right)\right)$

Lemma $2 M_{a}\left(\mathcal{A}\left(A_{q, q}\right)\right)$ contains Blocking Sets.

Proof. Let us remark that $M_{a}\left(\mathcal{A}\left(A_{q, q}\right)\right)$ contains $q$ ! points and $(q-1)$ ! lines with the same direction $(1, \ldots, 1)$ (by lemma 1). It follows, trivially, that the set given by one point for any line is a Blocking set.

Proof proposition [3. As consequence of lemma 2 and theorem 3 the complement $M_{a}\left(\mathcal{A}\left(A_{n, q}\right)\right)$ contains Blocking sets if $n \leq q$.

Moreover $P G(1, q)$ contains blocking sets. Then the statement follows from corollary 1 .

Remark 1 Let $M(\mathcal{A})$ be the complement of $\mathcal{A}$ in $P G(n, q)(A G(n, q))$ and $V$ a subspace of $M(\mathcal{A})$ without Blocking Sets. Then by proposition 1 it follows that $M(\mathcal{A})$ doesn't contain Blocking Sets. 
Remark 2 This new theory on Blocking Sets gives rise to a lot of questions and problems:

- how we can characterize a minimal unblocking arrangement in $P G(n, q)$ (or in $A G(n, q)$ ), i.e. an arrangement $\mathcal{A}$ such that $P G(n, q)$ doesn't contain Blocking Sets while $M(\mathcal{A})$ does and such that $\mathcal{A}$ is minimal with respect to the cardinality.

- Vice versa, how we can characterize a minimal blocking arrangement, i.e. an arrangement $\mathcal{A}$ such that $P G(n, q)$ contains Blocking Sets while $M(\mathcal{A})$ doesn't and such that $\mathcal{A}$ is minimal with respect to the cardinality.

An example of a minimal blocking arrangement is the line to infinity in $P G(2,3)$. Indeed it is know that $P G(2,3)$ contains Blocking Sets while $A G(2,3)$ doesn't.

- Other interesting questions are:

1. which is the link between the classical Blocking Sets theory and the theory on the complement $M(\mathcal{A})$ of an arrangement $\mathcal{A}$ ?

2. Which is the link between the theory of arrangements on finite spaces and the theory of blocking sets in the complement $M(\mathcal{A})$ of an arrangement $\mathcal{A}$ ? 


\section{References}

[1] A. Beutelspacher and F. Eugeni. On blocking sets in projective and affine spaces of large order. Communicated in Oberwolfach, Rend.Mat., 4:3-19, 1989.

[2] R. Bose and R. Burton. A characterization of Flat Spaces in a Finite Geometry and the uniqueness of the Hamming and the MacDonald Codes. J. Comb. Th., 1:96-104, 1966.

[3] A. Bruen. Blocking sets in projective plan. Siam. J.Appl.Math, 21:380-392, 1971.

[4] A. Bruen and J. Thas. Blocking sets. Geometriae Dedicata, 6:193-203, 1977.

[5] A. Cossidente, A. Gács, Cs. Mengyán, A. Siciliano, T. Sz onyi, and Z. Weiner. On large minimal blocking sets in PG(2,q). J.Comb. Des , 1:25-41, 2005.

[6] A. Gács, T. Sz onyi, and Zs. Weiner. On the spectrum of minimal blocking sets in PG(2,q). J. of Geometry , 76:256-281, 2003.

[7] J.W.P. Hirschfeld. Projective geometries over finite fields. Claredon Press, Oxford, 1998.

[8] S. Innamorati. The non existence of certain large minimal blocking sets . Mitt. Math. Sem. Giessen , 235:1-23, 1998.

[9] S. Innamorati and A. Maturo. The spectrum of minimal blocking sets . Discrete Math. , 208/209:339-347, 1999.

[10] F. Mazzocca and G. Tallini. On the nonexistence of blocking sets in PG $(n, q)$ and $A(n, q)$ for all large enough $n$. Simon Stevin, 1:43-50, 1985.

[11] P. Orlik and H. Terao. Arrangements of Hyperplanes, volume 300. SpringerVerlag, 1992. 